\title{
Inpatient Inherited Thrombophilia Testing
}

\author{
Christopher M. Petrilli, $\mathrm{MD}^{1 *}{ }^{\dagger}$, Lauren Heidemann, $\mathrm{MD}^{1 \dagger}$, Megan Mack, MD ${ }^{1,2}$, Paul Durance, PhD³, Vineet Chopra, MD, MSc ${ }^{1,2}$
}

${ }^{1}$ Department of Medicine, Division of General Internal Medicine, University of Michigan, Ann Arbor, Michigan; 2VA Ann Arbor Healthcare System, Ann Arbor, Michigan; ${ }^{3}$ Department of Quality Improvement Operations, University of Michigan, Ann Arbor, Michigan.

The "Things We Do for No Reason" (TWDFNR) series reviews practices which have become common parts of hospital care but which may provide little value to our patients. Practices reviewed in the TWDFNR series do not represent "black and white" conclusions or clinical practice standards, but are meant as a starting place for research and active discussions among hospitalists and patients. We invite you to be part of that discussion.

Inherited thrombophilia refers to a genetic condition that predisposes to an increased risk of venous thromboembolism (VTE). This disorder is prevalent in approximately $7 \%$ of the population and includes mutations such as factor $\mathrm{V}$ Leiden, prothrombin 20210, protein C deficiency, protein S deficiency, antithrombin deficiency, and methylene tetrahydrofolate reductase. The relative risk of VTE is 3- to 20-fold greater in patients with inherited thrombophilia compared with the general population. Is testing for inherited thrombophilia recommended? The available evidence suggests that testing for inherited thrombophilia is not recommended in most clinical settings. In patients without a personal history of VTE, thrombophilia results do not change management, as there is no evidence to support thromboprophylaxis in this setting. In patients with a personal history of provoked or unprovoked VTE, inpatient testing is not indicated, as results do not influence management, testing is not cost-effective, and a positive test result may lead to unnecessary patient anxiety or may result in unnecessary involvement of consultants. Testing in hospitalized patients has even more limitations because many thrombophilia tests are inaccurate in the setting of acute VTE and/or anticoagulation.

\section{CASE PRESENTATION}

A 23-year-old man presents to the emergency room with pleuritic chest pain and new oxygen requirement of $2 \mathrm{~L}$ nasal cannula. He has a history of unprovoked

*Address for correspondence and reprint requests: Christopher Petrilli, MD, 1500 East Medical Center Drive, Ann Arbor, Ml 48109; Telephone: 734-936-4385; Fax: 877-992-3793; E-mail: cpetrill@umich.edu

Additional Supporting Information may be found in the online version of this article.

${ }^{\dagger}$ Christopher and Lauren contributed equally to this work.

Received: January 21, 2016; Revised: April 27, 2016; Accepted: April 29, 2016

2016 Society of Hospital Medicine DOI 10.1002/jhm.2616

Published online in Wiley Online Library (Wileyonlinelibrary.com). lower extremity deep venous thrombosis (DVT) diagnosed at age 20 and completed 3 months of systemic anticoagulation without complications. He reports no family history of clotting disorders or venous thromboembolism (VTE) and no reversible risk factors for VTE such as prolonged immobility, recent surgery, or high-risk medications. A computed tomogram pulmonary embolism protocol shows multiple right lower lobe, segmental pulmonary emboli. Anticoagulation is initiated, and the patient is admitted to the hospital. Will inpatient inherited thrombophilia testing impact management for this case?

\section{WHY MAY INHERITED THROMBOPHILIA TESTING PROVE HELPFUL?}

The annual incidence rate of a first VTE event is estimated as 117 per 100,000 individuals per year. ${ }^{1}$ The most common presentations are symptomatic DVT of the leg (annual incidence approximately 48 per 100,000 people), or a pulmonary embolism (annual incidence approximately 69 per 100,000 people). ${ }^{1}$ Pulmonary embolism results in death in up to $30 \%$ of untreated patients and $2.5 \%$ of patients who receive systemic anticoagulation. ${ }^{2}$ Principal in the pathogenesis of VTE are factors described by Virchow's triad: venous stasis, endothelial injury, and systemic hypercoagulability. By identifying a mutation in 1 or more of the factors in the clotting pathway, an evaluation for inherited thrombophilia theoretically may unearth factors that drive systemic hypercoagulability and inform decision making so as to prevent future events.

Inherited thrombophilia refers to a genetic condition that predisposes to an increased risk of VTE. ${ }^{3}$ Approximately $7 \%$ of the general population has inherited thrombophilia, which includes factor V Leiden (FVL) mutation, prothrombin 20210 mutation (PT20210), protein C deficiency, protein S deficiency, antithrombin III (ATIII) deficiency, and methylene tetrahydrofolate reductase mutation (MTHFR). ${ }^{4}$ Of note, the definition does not include acquired etiologies, such as antiphospholipid antibody syndrome. Depending on the underlying condition and expression of the genetic abnormality, the relative risk of VTE in patients with inherited thrombophilia is 3- to 20-fold greater than that of the general population. ${ }^{5}$ Therefore, it is logical to consider that testing for inherited thrombophilia might be clinically useful. However, the evidence for doing so is very limited. 
TABLE 1. Limitations of Thrombophilia Workup in the Setting of Acute VTE or Anticoagulation

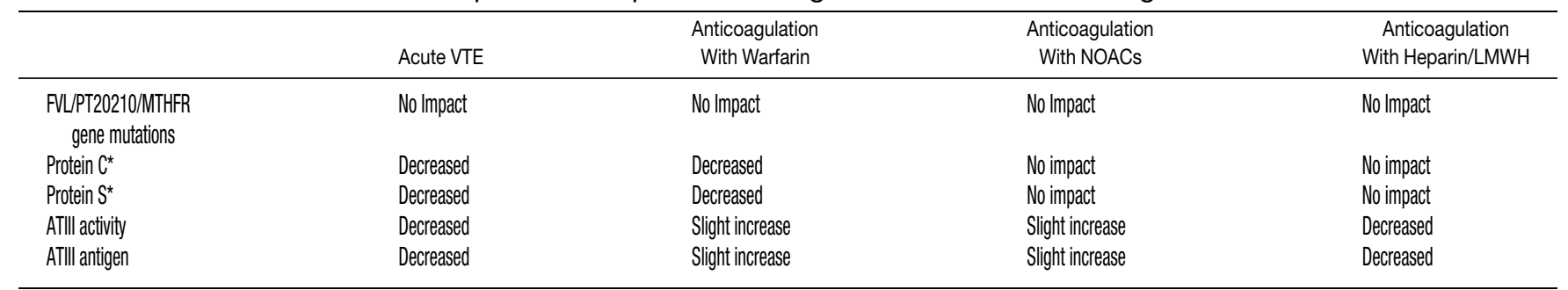

NOTE: Abbreviations: ATIII, antithrombin III deficiency; FVL, factor V Leiden gene mutation; LMWH, Low-molecular-weight heparin; MTHFR, methylenetetrahydrofolate reductase gene mutation; NOACs, novel oral anticoagulants (anti-Xa or direct thrombin inhibitors); PT20210, prothrombin 20210 gene mutation; VTE, venous thromboembolism. . Deficiency in both protein and functional assays.

\section{DOES INHERITED THROMBOPHILIA TESTING CHANGE MANAGEMENT?}

An inherited thrombophilia evaluation is unlikely to affect management in most clinical settings. There is no current evidence to support primary prophylaxis ${ }^{6}$ nor is there evidence that management of patients with recurrent VTE should be altered in the setting of inherited thrombophilia.

To date, no prospective trials have evaluated the efficacy of anticoagulant use for primary prevention of VTE in patients with inherited thrombophilia. ${ }^{6}$ Given the limited evidence for thromboprophylaxis and risks of anticoagulation, primary prevention for patients with inherited thrombophilia that remain asymptomatic is not recommended by the current American College of Chest Physicians guidelines. ${ }^{7,8}$

Similarly, in patients with a first VTE or recurrent VTE, diagnosis of inherited thrombophilia is often not associated with recurrent events, which suggests that other nongenetic factors may be just as important, if not more important, in determining the risk of recurrence. ${ }^{9}$ Although no randomized controlled or controlled clinical trials have evaluated the effects of testing for inherited thrombophilia on recurrent VTE, ${ }^{10,11}$ several prospective studies have assessed risk factors for recurrence. Data from these studies suggest that recurrence rates after unprovoked VTE are only weakly correlated with inherited thrombophilia status. ${ }^{12,13}$ Rather, it is postulated that patients with recurrent VTE may exhibit a prothrombotic tendency regardless of underlying genetic predisposition. In this case, decisions regarding anticoagulation do not vary by thrombophilia status. Instead, thrombophilia testing may divert attention away from the management of more prevalent, potentially modifiable risk factors such as immobility, oral contraceptive use, or malignancy, all of which are associated with recurrent VTE. ${ }^{14}$ These provoking factors are the most important determinants of the chance of VTE recurrence as well as the most significant factors to take into account when deciding duration of anticoagulation.

Christiansen et al. performed a prospective study evaluating the association between recurrent VTE and thrombophilia status. After following 474 patients with confirmed first episode VTE for a mean of 7.3 years, no statistically significant risk of VTE was found for patients with FVL (hazard ratio [HR]: 1.2, 95\% confidence interval [CI]: 0.7-1.9), PT20210 (HR: 0.7, 95\% CI: 0.3-2.0), or an anticoagulant (protein C, protein $\mathrm{S}$ or ATIII) deficiency (HR: 1.8, 95\% CI: 0.93.7). ${ }^{15}$ Although unexplained VTE was statistically associated with VTE recurrence, heritable thrombophilia status was not.

In a systematic review and meta-analysis investigating the association of FVL and PT20210 with recurrent VTE, Ho and colleagues found a statistically significant risk of recurrent VTE in patients with inherited thrombophilia due to FVL (odds ratio [OR]: 1.41, 95\% CI: 1.14-1.75) and PT20210 (OR: 1.72, 95\% CI: 1.272.31), and reported that "at most, only up to 1 in 6 recurrent VTEs may be attributable to these mutations." 16 Based on this relatively modest effect, the authors question the utility of testing for inherited thrombophilia, as thrombophilia status is unlikely to warrant a change in type or duration of treatment.

Regardless of whether an underlying inherited thrombophilia is identified, patients with history of recurrent VTE are often candidates for long-term anticoagulation. Testing for inherited thrombophilia in patients with prior VTE events will therefore not influence decisions regarding clinical management. Additionally, such testing may be confounded by ongoing disease or treatment (Table 1). For example, protein C, protein $\mathrm{S}$ antigen, and ATIII levels are low in the setting of acute VTE. ${ }^{17,18}$ Likewise, protein $\mathrm{C}$ and $\mathrm{S}$ (vitamin $\mathrm{K}$-dependent proteins) will be low in the setting of anticoagulation with warfarin. ${ }^{19}$ Moreover, ATIII activity and antigen levels are low in the setting of heparin use. ${ }^{20}$ Lack of provider awareness regarding these interactions may have important negative consequences, including a spurious diagnosis of thrombophilia, ${ }^{21,22}$ unnecessary hematology consultation, and psychological distress to patients in the form of ongoing unwarranted testing or apprehension regarding recurrence. ${ }^{23}$

Additionally, this expensive evaluation has estimated direct costs of $\$ 1100$ to $\$ 2400$ per thrombophilia panel based on estimation of charges billed by a large commercial laboratory. ${ }^{24,25}$ In 2014, over 280,000 claims were submitted under Medicare Part 
B across all care settings for a thrombophilia analysis including FVL, PT20210, and MTHFR gene mutations, ${ }^{24}$ which would equate to between $\$ 300$ million to $\$ 672$ million. ${ }^{26}$ Unfortunately, there have been no large-scale trials to assess cost-effectiveness. However, the Evaluation of Genomic Applications in Practice and Prevention (EGAPP) Working Group stated that "cost-effectiveness modeling studies in this area require updating with current VTE risk estimates but are suggestive that routine FVL/PT20210 testing is not cost-effective." 27

\section{ARE THERE CIRCUMSTANCES IN WHICH INPATIENT INHERITED THROMBOPHILIA TESTING PROVES BENEFICIAL?}

The evidence for when to test for inherited thrombophilia is very limited and is often based on individualized risk. The current EGAPP guidelines acknowledge this limitation, specifically noting that there is a paucity of data evaluating management or prophylaxis of patients with homozygous or compound heterozygous FVL or P20210 mutation, and a lack of data surrounding whether or not knowledge of thrombophilia mutation should affect anticoagulation treatment. ${ }^{27}$ This is why an individualized approach is deemed necessary. For example, the decision to prescribe hormone replacement therapy in women with a family history of inherited thrombophilia may be better informed by testing prior to treatment. Similarly, pregnant women with a family history or personal history of VTE may also benefit from inherited thrombophilia testing, as this may influence antepartum or postpartum management. ${ }^{28,29}$ The National Institute for Health and Clinical Excellence (NICE) guidelines recommend consideration of testing for hereditary thrombophilia in patients with unprovoked VTE and a first-degree relative with VTE, if stopping anticoagulation treatment is planned; however, these recommendations are based solely on Guideline Development Group's experience and opinion. ${ }^{30}$ Regardless, testing for inherited thrombophilia has significant potential consequences. Patients at risk should meet with an outpatient hematologist and/or a genetic counselor, if available, to determine the risks and benefits of testing.

\section{WHAT DO GUIDELINES SAY ABOUT INHERITED THROMBOPHILIA TESTING?}

The most recent NICE guidelines recommend against offering inherited thrombophilia testing to patients presenting with a provoked VTE in any clinical setting. ${ }^{30}$ In patients diagnosed with unprovoked VTE, testing should not be considered unless a first degree relative with a history of VTE exists. ${ }^{30}$ The NICE guidelines also recommend against routinely offering thrombophilia testing to asymptomatic first-degree relatives of patients with a history of VTE or known inherited thrombophilia. This recommendation is reflected in the American Society of Hematology's Choosing Wisely rec- ommendations since 2013. ${ }^{31}$ Further, The American College of Medical Genetics and Genomics' Choosing Wisely recommendations from 2015 state that MTHFR mutations should never be included in any thrombophilia workup, as "recent meta-analyses have disproven an association between the presence of these variants and venous thromboembolism." 32

The EGAPP Working Group recommends against routine testing for FVL or PT20210 in patients who present with an idiopathic VTE, as longer-term anticoagulation offers similar benefits to patients with or without these mutations. ${ }^{27}$ EGAPP also recommends against testing asymptomatic adult family members of patients with VTE and/or an FVL or PT20210 mutation for the purpose of considering primary prophylactic anticoagulation. In these circumstances, it is felt that the potential risks of thrombophilia testing outweigh any potential benefits.

\section{HOW SHOULD HOSPITALISTS APPROACH TESTING OF INHERITED THROMBOPHILIA?}

The providers in our case presentation are challenged with determining whether inpatient thrombophilia evaluation will add value to the evaluation of patients with unprovoked VTE. The available evidence suggests that clinicians should avoid ordering thrombophilia testing for hospitalized patients with unprovoked VTE because (1) many thrombophilia tests are inaccurate in the setting of acute VTE and/or anticoagulation, (2) results of testing often do not influence management, (3) testing is not cost-effective, (4) a positive test result may lead to unnecessary patient anxiety, and (5) testing may result in inappropriately prolonged anticoagulation courses or unnecessary involvement of inpatient consultants. For these reasons, the patient in our case presentation should not be tested for inherited thrombophilia. In patients with personal or family histories of recurrent thromboembolism, modifiable clinical risk factors should be addressed, as these are more likely to influence treatment decisions compared to genetic testing. Finally, patients may be referred to an outpatient hematologist or geneticist for individualized discussions of risks and benefits of testing for inherited thrombophilia.

\section{CONCLUSION}

Inpatient evaluation for inherited thrombophilia for VTE is not clinically useful, cost-effective, or reliable in the setting of VTE. The result of such testing does not affect management of acute primary or recurrent VTE. Testing should only be considered using an individualized approach in the outpatient setting with appropriate genetic counseling.

Disclosure: Christopher M. Petrilli, MD, and Lauren Heidemann, MD, contributed equally to this work. The authors report no conflicts of interest.

Do you think this is a low-value practice? Is this truly a "Thing We Do for No Reason?" Share what you do in your practice and join in the 
conversation online by retweeting it on Twitter (\#TWDFNR) and liking it on Facebook. We invite you to propose ideas for other "Things We Do for No Reason" topics by emailing TWDFNR@hospitalmedicine. org.

\section{References}

1. Silverstein MD, Heit JA, Mohr DN, Petterson TM, O'Fallon WM, Melton LJ III. Trends in the incidence of deep vein thrombosis and pulmonary embolism: a 25-year population-based study. Arch Intern Med. 1998;158(6):585-593.

2. Carson JL, Kelley MA, Duff A, et al. The clinical course of pulmonary embolism. N Engl J Med. 1992;326(19):1240-1245.

3. Khan S, Dickerman JD. Hereditary thrombophilia. Thromb J. 2006;4: 15.

4. Lensing AW, Prandoni P, Prins MH, Buller HR. Deep-vein thrombosis. Lancet. 1999;353(9151):479-485.

5. Barbar S, Noventa F, Rossetto V, et al. A risk assessment model for the identification of hospitalized medical patients at risk for venous thromboembolism: the Padua Prediction Score. J Thromb Haemost. 2010;8(11):2450-2457.

6. Bates SM, Greer IA, Middeldorp S, et al. VTE, thrombophilia, antithrombotic therapy, and pregnancy: Antithrombotic Therapy and Prevention of Thrombosis, 9th ed: American College of Chest Physicians Evidence-Based Clinical Practice Guidelines. Chest. 2012; 141(2 suppl):e691S-e736S.

7. De Stefano V, Rossi E. Testing for inherited thrombophilia and consequences for antithrombotic prophylaxis in patients with venous thromboembolism and their relatives. A review of the Guidelines from Scientific Societies and Working Groups. Thromb Haemost. 2013; 110(4):697-705.

8. Kahn SR, Lim W, Dunn AS, et al. Prevention of VTE in nonsurgical patients: antithrombotic therapy and prevention of thrombosis, 9th ed: American College of Chest Physicians Evidence-Based Clinical Practice Guidelines. Chest. 2012;141(2 suppl):e195S-e226S.

9. Segal JB, Brotman DJ, Necochea AJ, et al. Predictive value of factor V Leiden and prothrombin G20210A in adults with venous thromboembolism and in family members of those with a mutation: a systematic review. JAMA. 2009;301(23):2472-2485.

10. Cohn D, Vansenne F, de Borgie C, Middeldorp S. Thrombophilia testing for prevention of recurrent venous thromboembolism. Cochrane Database Syst Rev. 2009;(1):CD007069.

11. Cohn DM, Vansenne F, de Borgie CA, Middeldorp S. Thrombophilia testing for prevention of recurrent venous thromboembolism. Cochrane Database Syst Rev. 2012;12:CD007069.

12. Baglin T, Luddington R, Brown K, Baglin C. Incidence of recurrent venous thromboembolism in relation to clinical and thrombophilic risk factors: prospective cohort study. Lancet. 2003;362(9383):523526.

13. Kearon C, Julian JA, Kovacs MJ, et al. Influence of thrombophilia on risk of recurrent venous thromboembolism while on warfarin: results from a randomized trial. Blood. 2008;112(12):4432-4436.

14. Merriman L, Greaves M. Testing for thrombophilia: an evidencebased approach. Postgrad Med J. 2006;82(973):699-704.

15. Christiansen SC, Cannegieter SC, Koster T, Vandenbroucke JP, Rosendaal FR. Thrombophilia, clinical factors, and recurrent venous thrombotic events. JAMA. 2005;293(19):2352-2361.
16. Ho WK, Hankey GJ, Quinlan DJ, Eikelboom JW. Risk of recurrent venous thromboembolism in patients with common thrombophilia: a systematic review. Arch Intern Med. 2006;166(7):729-736.

17. Vigano D'Angelo S, Comp PC, Esmon CT, D'Angelo A. Relationship between protein $\mathrm{C}$ antigen and anticoagulant activity during oral anticoagulation and in selected disease states. J Clin Invest. 1986;77(2): 416-425.

18. Patnaik MM, Moll S. Inherited antithrombin deficiency: a review. Haemophilia. 2008;14(6):1229-1239.

19. Weiss P, Soff GA, Halkin H, Seligsohn U. Decline of proteins C and S and factors II, VII, IX and X during the initiation of warfarin therapy. Thromb Res. 1987;45(6):783-790.

20. Heit JA. Thrombophilia: common questions on laboratory assessment and management. Hematology Am Soc Hematol Educ Program. 2007:127-135.

21. Kadauke S, Khor B, Van Cott EM. Activated protein C resistance testing for factor V Leiden. Am J Hematol. 2014;89(12):1147-1150.

22. Fair DS, Revak DJ. Quantitation of human protein $S$ in the plasma of normal and warfarin-treated individuals by radioimmunoassay. Thromb Res. 1984;36(6):527-535.

23. Bank I, Scavenius MP, Buller HR, Middeldorp S. Social aspects of genetic testing for factor V Leiden mutation in healthy individuals and their importance for daily practice. Thromb Res. 2004;113(1):7-12.

24. Abramson N, Abramson S. Hypercoagulability: clinical assessment and treatment. South Med J. 2001;94(10):1013-1020.

25. Somma J, Sussman, II, Rand JH. An evaluation of thrombophilia screening in an urban tertiary care medical center: A "real world" experience. Am J Clin Pathol. 2006;126(1):120-127.

26. CodeMap. Available at: https://www.codemap.com. Accessed January $18,2016$.

27. Evaluation of Genomic Applications in Practice and Prevention (EGAPP) Working Group. Recommendations from the EGAPP Working Group: routine testing for Factor V Leiden (R506Q) and prothrombin (20210G $>$ A) mutations in adults with a history of idiopathic venous thromboembolism and their adult family members. Genet Med. 2011;13(1):67-76.

28. Brill-Edwards P, Ginsberg JS, Gent M, et al. Safety of withholding heparin in pregnant women with a history of venous thromboembolism. Recurrence of Clot in This Pregnancy Study Group. N Engl J Med. 2000;343(20):1439-1444.

29. Friederich PW, Sanson BJ, Simioni P, et al. Frequency of pregnancyrelated venous thromboembolism in anticoagulant factor-deficient women: implications for prophylaxis. Ann Intern Med. 1996;125(12): 955-960.

30. Chong LY, Fenu E, Stansby G, Hodgkinson S; Guideline Development Group. Management of venous thromboembolic diseases and the role of thrombophilia testing: summary of NICE guidance. BMJ. 2012; 344:e3979.

31. American Society of Hematology. Ten things physicians and patients should question. Choosing Wisely website. Available at: http://www. choosingwisely.org/societies/american-society-of-hematology. Published December 4, 2013. Accessed January 18, 2016.

32. American College of Medical Genetics and Genomics. Five Things patients and providers should question. Choosing Wisely website. Available at: http://www.choosingwisely.org/societies/american-college-of-medical-genetics-and-genomics. Published July 10, 2015. Accessed March 13, 2016. 